Article

\title{
Bismuth triflate: A highly efficient catalyst for the synthesis of bio-active coumarin compounds via one-pot multi-component reaction
}

\author{
Mahmoud. Abd El Aleem. Ali. Ali. El-Remaily* \\ Department of Chemistry, Faculty of Science, Sohag University-82524, Sohag, Egypt
}

\section{A R T I C L E I N F O}

Article history:

Received 9 January 2015

Accepted 2 February 2015

Published 20 July 2015

\section{Keywords:}

Coumarin

Chalcone

Pyrazoline

One-pot multi-component reaction

Salicylaldehyde

$\alpha$-Ketoester

Bismuth triflate $\left[\mathrm{Bi}(\mathrm{OTf})_{3}\right]$

\section{A B S T R A C T}

A series of coumarin-chalcone hybrid compounds and coumarins linked to pyrazoline was synthesized in good yield and short time using a simple and efficient method. This method involved the one-pot reaction of salicylaldehyde, an $\alpha$-ketoester and an aromatic aldehyde (in the case of the coumarin-chalcone derivatives) in addition to hydrazine hydrate (in the case of the pyrazolyl coumarins) in the presence of a catalytic amount of bismuth triflate [Bi(OTf $)_{3}, 5 \mathrm{~mol} \%$ ]. The synthesized compounds showed scavenging activity towards the free radical 2,2-diphenyl-1-picrylhydrazyl. All compounds were characterized using IR, ${ }^{1} \mathrm{H}$ NMR and ${ }^{13} \mathrm{C}$ NMR spectroscopy.

(C) 2015, Dalian Institute of Chemical Physics, Chinese Academy of Sciences. Published by Elsevier B.V. All rights reserved.

\section{Introduction}

The coumarin moiety is an important structural motif in natural products and highly bioactive compounds. Coumarin-containing compounds exhibit broad biological activity with, for example, antioxidant, anticoagulant, antifungal, anthelmintic, cytotoxic or hypnotic properties [1-10]. Because of their fluorescent properties, coumarins are also widely applied as agrochemicals; used in optical brighteners, cosmetics and food; and employed as scattered fluorescent and disciplined laser-dye optical agents [11,12]. Chalcones, one of the major classes of natural products prevalent in fruits, vegetables, spices and soy-based foodstuffs, have been reported to have several biological properties $[13,14]$. The chalcone moiety imparts biological activity upon the molecule, giving it, for example, anti-inflammatory [15], antifungal [16], antioxidant [17], anti- malarial [18], antituberculosis [19], analgesic [20], anti-HIV [21] and antitumor [22] properties. Linking coumarins to pyrazoline rings may lead to compounds with important properties, as pyrazoline itself shows anti-inflammatory, antidiabetic, anesthetic, analgesic, and other potent selective activity, such as behavior as a nitric oxide synthase (NOS) inhibitor or a cannabinoid CB1 receptor antagonist [23].

Multi-component reactions (MCRs) are processes in which three or more reactants are joined in a single step to produce products that combine substantial portions of all the reactants [24-27]. In recent years, MCRs have unfolded as a powerful tool for creating the substructures of novel and complex molecules due to their advantages over traditional multi-step synthesis. The major advantages of MCRs include their lower cost, shorter reaction time and high atom economy, lower energy requirements, and the avoidance of time consuming and ex-

\footnotetext{
* Corresponding author. Tel: +201143627127; Fax: +2093601159; E-mail: msremaily@yahoo.com 
pensive purification processes. MCRs are generally more eco-friendly than traditional syntheses, and offer fast access to wide libraries of compounds with different functionalities [28-32]. During recent years, Bi(III) salts have caught the attention of synthetic organic chemists as effective catalysts because of their low toxicity, eco friendliness, ease of handling, low cost and relative insensitivity to air and moisture [33-38]. Among the $\mathrm{Bi}(\mathrm{III})$ salts, bismuth triflate $\left[\mathrm{Bi}\left(\mathrm{OTf}_{3}\right]_{3}\right.$ is the most efficient catalyst, and is particularly attractive because it is commercially available or easily prepared from commercially available starting materials [39-46].

One of my aims in recent years has been the development of new tools and methodologies for the synthesis of heterocyclic compounds using an efficient catalyst. Following this aim I designed hybrid molecules based on naturally occurring compounds, coumarin-chalcone moieties and coumarines linked to pyrazolines, using multi-component reaction techniques in the presence of a catalytic amount (5 mol\%) of the green catalyst Bi(OTf) 3 .

\section{Experimental}

All melting points were recorded on a Melt-Temp II melting point apparatus. IR spectra were measured as $\mathrm{KBr}$ pellets on a Shimadzu DR-8001 spectrometer. ${ }^{1} \mathrm{H}$ NMR spectra were recorded on a Bruker DRX 300 or $400 \mathrm{MHz}$ spectrometer using TMS as an internal reference and DMSO-d6 or $\mathrm{CDCl}_{3}$ as the solvent. The purity of all compounds was checked on thin layer chromatography (TLC) plates. All the synthesized compounds were prepared according to the method described by Sugino et al. [47] and Bhatnagar et al. [48]. Confirmation of the proposed structures of all prepared compounds by IR, ${ }^{1} \mathrm{HNMR}$ and ${ }^{13} \mathrm{C}$ NMR spectroscopy are also reported.

\subsection{General procedure for the synthesis of 3-cinnamoyl coumarins (5a-5n)}

\section{Method A}

In a $50 \mathrm{~mL}$ round bottom flask, salicylaldehyde 1 ( $1 \mathrm{mmol})$, methyl acetoacetate or ethyl acetoacetate 2 (1 mmol) and aromatic aldehyde 3 ( $1 \mathrm{mmol}$ ) were mixed in the presence of Bi(OTf)3 ( $5 \mathrm{~mol} \%$ ) in dichloromethane (DCM, $5 \mathrm{~mL}$ ). The reaction mixture was stirred at $50{ }^{\circ} \mathrm{C}$ for a specified time. The progress of the reaction was monitored by TLC. The products precipitated rapidly after 15 to $30 \mathrm{~min}$ (see Table 2). The solid material was filtered off, washed with water, dried, and recrystallized from ethanol to furnish pure 3-cinnamoylcoumarin derivatives (5a-5n).

\section{Method B}

\section{First step: Synthesis of 3-acetylcoumarin (4)}

A mixture of salicylaldehyde (1 eq.), methyl acetoacetate or ethyl acetoacetate (1 eq.) and a few drops of piperidine were stirred at room temperature without any solvent. Precipitation occurred immediately. The compound was then recrystallized from ethanol [47]. Yield 89\%; M.P. $119-121{ }^{\circ} \mathrm{C}$; ${ }^{1} \mathrm{H}$ NMR (300 $\left.\mathrm{MHz}_{\mathrm{CDCl}}, \delta / \mathrm{ppm}\right) 8.34(\mathrm{~s}, 1 \mathrm{H}), 7.54-7.42(\mathrm{~m}, 2 \mathrm{H}), 7.26-7.12$ (m, 2H), $2.56(\mathrm{~s}, 3 \mathrm{H})$.

\section{Second step: Synthesis of 5a-5n}

A mixture of 3-acetylcoumarin 4 (1 eq.) and the corresponding aldehyde (1.2 eq.) were mixed in the presence of $\mathrm{Bi}(\mathrm{OTf})_{3}(5 \mathrm{~mol} \%)$ in DCM $(5 \mathrm{~mL})$. The reaction mixture was stirred at $50{ }^{\circ} \mathrm{C}$ for a specified time. The progress of the reaction was monitored by TLC. The products precipitated slowly after $4 \mathrm{~h}$. The solid material was filtered off, washed with water, dried, and recrystallized from ethanol.

1-(3'-Coumarinyl)-3-phenyl-2-propen-1-one (5a). Yield 89\%; M.P. $180-182{ }^{\circ} \mathrm{C}$; IR ( $\mathrm{KBr}, \mathrm{cm}^{-1}$ ): 3037 (Ar, C-H str), 2968 (alkane, C-H str), 1714 (C=0), 1683 (C=0), 1610 (C=C); ${ }^{1} \mathrm{H}$ NMR $\left(\mathrm{CDCl}_{3}, \delta / \mathrm{ppm}\right): 8.61(\mathrm{~s}, 1 \mathrm{H}, \mathrm{C}-\mathrm{H}), 7.90(\mathrm{~d}, 1 \mathrm{H},=\mathrm{CH}), 7.71-7.54$ (m, 4H, coumarin), 7.419-7.266 (m, 5H, Ar-H), $6.87(\mathrm{~d}, 1 \mathrm{H}$, $=\mathrm{C}-\mathrm{H}) ;{ }^{13} \mathrm{C} \mathrm{NMR}\left(\mathrm{CDCl}_{3}, \delta / \mathrm{ppm}\right): 196.52,161.23,153.22$, 147.36, 138.69, 133.25, 132.00, 130.78, 129.89, 129.14, 128.55, $127.19,124.74,124.10,122.05,114.36$; Anal. Calc. for $\mathrm{C}_{18} \mathrm{H}_{12} \mathrm{O}_{3}$ (276.29): C 78.25\%, H 4.38\%; Found: C 78.17\%, H 4.28\%.

1-(3'-Coumarinyl)-3-(4"-methoxyphenyl)-2-propen-1-one (5b). Yield 91\%; M.P. 140-142 ${ }^{\circ} \mathrm{C}$; IR (KBr, cm$\left.{ }^{-1}\right): 3052(\mathrm{Ar}$, C-H str), 1705 (C=0), 1681 (C=0), 1604 (C=C str), 1612 (C=C ); ${ }^{1} \mathrm{H}$ NMR ( $\left.\mathrm{CDCl}_{3}, \delta / \mathrm{ppm}\right): 8.54(\mathrm{~s}, 1 \mathrm{H}, \mathrm{C}-\mathrm{H}), 7.80(\mathrm{~d}, 1 \mathrm{H},=\mathrm{C}-\mathrm{H})$, 7.68-7.63 (m, 4H, coumarin), 7.44-7.21(m, 4H, Ar-H), $6.91(\mathrm{~d}$, $1 \mathrm{H},=\mathrm{C}-\mathrm{H}), 2.74\left(\mathrm{~s}, 3 \mathrm{H}, \mathrm{O}-\mathrm{CH}_{3}\right) ;{ }^{13} \mathrm{C} \mathrm{NMR}\left(\mathrm{CDCl}_{3}, \delta / \mathrm{ppm}\right)$ : 190.12, 162.20, 161.54, 154.79, 147.30, 138.89, 131.88, 130.07, 130.78, 128.84, 128.04, 127.14, 124.80, 124.09, 122.08, 114.36, 55.20; Anal. Calc. for $\mathrm{C}_{19} \mathrm{H}_{14} \mathrm{O}_{4}$ (306.32): C 74.50\%, H 4.61\%; Found: C 74.44\%, $\mathrm{H} 4.58 \%$.

1-(3'-Coumarinyl)-3-(4" -chlorophenyl)-2-propen-1-one (5c). Yield 96\%; M.P. 188-190 ${ }^{\circ} \mathrm{C}$; IR (KBr, cm-1): 3031 (Ar, C-H str), $1715(\mathrm{C}=0), 1679$ (C=0); $1610(\mathrm{C}=\mathrm{C}) ;{ }^{1} \mathrm{H}$ NMR ( $\mathrm{CDCl}_{3}$, $\delta / \mathrm{ppm}): 8.59$ (s, 1H, C-H), $7.98(\mathrm{~d}, 1 \mathrm{H},=\mathrm{C}-\mathrm{H}), 7.67-7.52(\mathrm{~m}, 4 \mathrm{H}$, coumarin), 7.41-7.26 (m, 4H, Ar-H), $6.84(\mathrm{~d}, 1 \mathrm{H},=\mathrm{C}-\mathrm{H}) ;{ }^{13} \mathrm{C}$ NMR ( $\left.\mathrm{CDCl}_{3}, \delta / \mathrm{ppm}\right): 194.50,162.20,154.77,147.47,138.78$, 135.54, 134.11, 130.78, 129.98, 129.41, 128.05, 127.84, 124.64, 124.09, 122.07, 114.62; Anal. Calc. for $\mathrm{C}_{18} \mathrm{H}_{11} \mathrm{O}_{3} \mathrm{Cl}$ (310.74): C 69.58\%, H 3.57\%, Cl 11.41\%; Found: C 69.55\%, H 3.59\%, Cl $11.09 \%$.

1-(3'-Coumarinyl)-3-(2" -chlorophenyl)-2-propen-1-one (5d). Yield 89\%; M.P. 204-206 ${ }^{\circ} \mathrm{C}$; IR (KBr, cm-1): 3054 (Ar, C-H str), 1721 (C=0), 1684(C=0), $1610(\mathrm{C}=\mathrm{C}) ;{ }^{1} \mathrm{H}$ NMR $\left(\mathrm{CDCl}_{3}\right.$, $\delta / \mathrm{ppm}): 8.61$ (s, 1H, C-H), $7.96(\mathrm{~d}, 1 \mathrm{H},=\mathrm{C}-\mathrm{H}), 7.70-7.65(\mathrm{~m}, 4 \mathrm{H}$, coumarin), 7.45-7.26 (m, 4H, Ar-H), $6.26(\mathrm{~d}, 1 \mathrm{H},=\mathrm{C}-\mathrm{H}) ;{ }^{13} \mathrm{C}$ NMR $\left(\mathrm{CDCl}_{3}, \delta / \mathrm{ppm}\right): 193.41,162.13,154.77,143.44,138.58$, 134.04, 133.57, 130.87, 130.08, 129.94, 128.76, 128.14, 127.84, $127.04,124.71,124.13,122.06,114.60 ;$ Anal. Calc. for $\mathrm{C}_{18} \mathrm{H}_{11} \mathrm{O}_{3} \mathrm{Cl}$ (310.74): C 69.58\%, $\mathrm{H} 3.57 \%, \mathrm{Cl} 11.41 \%$; Found: $\mathrm{C}$ 69.55\%, H 3.59\%, Cl 11.09\%.

1-(3'-Coumarinyl)-3-(4"-hydroxyphenyl)-2-propen-1-one (5e). Yield 91\%; M.P. $176-178{ }^{\circ} \mathrm{C}$; IR (KBr, cm-1): $3389(\mathrm{OH})$, 3038 (Ar, C-H), 1702 (C=0), 1689 (C=0), 1612 (C=C); ${ }^{1} \mathrm{H}$ NMR $\left(\mathrm{CDCl}_{3}, \delta / \mathrm{ppm}\right): 9.73(\mathrm{~s}, 1 \mathrm{H}, \mathrm{OH}), 8.67(\mathrm{~s}, 1 \mathrm{H}, \mathrm{C}-\mathrm{H}), 7.95(\mathrm{~d}, 1 \mathrm{H}$, $=\mathrm{C}-\mathrm{H}), 7.77-7.60(\mathrm{~m}, 4 \mathrm{H}$, coumarin), 7.50-7.32 (m, 4H, Ar-H), $6.86(\mathrm{~d}, 1 \mathrm{H},=\mathrm{C}-\mathrm{H}) ;{ }^{13} \mathrm{C} \mathrm{NMR}\left(\mathrm{CDCl}_{3}, \delta / \mathrm{ppm}\right): 189.18,162.38$, 161.74, 154.77, 147.32, 138.30, 131.54, 130.04, 128.17, 127.79, $127.09,124.64,124.09,122.07,116.41,114.62$; Anal. Calc. for $\mathrm{C}_{18} \mathrm{H}_{12} \mathrm{O}_{4}$ (292.29): C 73.97\%, H 4.14\%; Found: C 73.81\%, H $4.11 \%$. 
1-(3'-Coumarinyl)-3-(2"-hydroxyphenyl)-2-propen-1-one (5f). Yield 90\%; M.P. 78-79 ${ }^{\circ} \mathrm{C}$; IR (KBr, cm-1): 3412 (OH str), 3041 (Ar, C-H), 1712 (C=0), 1680 (C=0), 1610 (C=C); ${ }^{1} \mathrm{H}$ NMR $\left(\mathrm{CDCl}_{3}, \delta / \mathrm{ppm}\right): 9.67$ (s, 1H, OH), $8.76(\mathrm{~s}, 1 \mathrm{H}, \mathrm{C}-\mathrm{H}), 7.75(\mathrm{~d}, 1 \mathrm{H}$, =C $-\mathrm{H})$, 7.48-7.42 (m, 4H, coumarin), 7.10-6.93 (m, 4H, Ar-H), $6.64(\mathrm{~d}, 1 \mathrm{H},=\mathrm{C}-\mathrm{H}) ;{ }^{13} \mathrm{C} \mathrm{NMR}\left(\mathrm{CDCl}_{3}, \delta / \mathrm{ppm}\right): 191.47,162.10$, $154.79,143.04,138.78,134.14,133.47,130.37,130.08,129.91$, 128.74, 128.14, 127.84, 127.04, 124.71, 124.13, 122.06, 114.62; Anal. Calc. for $\mathrm{C}_{18} \mathrm{H}_{12} \mathrm{O}_{4}$ (292.29): C 73.97\%, H 4.14\%; Found: C 73.81\%, H 4.11\%.

1-(3'-Coumarinyl)-3-(2"-furyl)-2-propen-1-one (5g). Yield 89\%; M.P. 101-103 ${ }^{\circ} \mathrm{C}$; IR (KBr, cm-1): 3037 (Ar, C-H), 1716 $(\mathrm{C}=0), 1680(\mathrm{C}=0), 1608(\mathrm{C}=\mathrm{C}) ;{ }^{1} \mathrm{H}$ NMR $\left(\mathrm{CDCl}_{3}, \delta / \mathrm{ppm}\right): 8.58$ (d, 1H, =C-H), $7.83(\mathrm{~d}, 1 \mathrm{H},=\mathrm{C}-\mathrm{H}), 7.70-7.60$ (m, 4H, coumarin), 7.45-7.34 (m, 3H, furyl), $6.85(\mathrm{~d}, 1 \mathrm{H},=\mathrm{C}-\mathrm{H}) ;{ }^{13} \mathrm{C} \mathrm{NMR}\left(\mathrm{CDCl}_{3}\right.$, $\delta / \mathrm{ppm}): 188.40,162.65,154.79,151.32,145.00,138.84$, 133.40, 130.07, 127.69, 125.61, 125.04, 124.54, 122.44, 120.32, 114.74, 106.47; Anal. Calc. for $\mathrm{C}_{16} \mathrm{H}_{10} \mathrm{O}_{4}$ (266.25): C 72.18\%, H 3.79\%; Found: C 72.01\%, H 3.61\%.

1-(3'-Coumarinyl)-3-(2",4",6"-trimethoxyphenyl)-2-propen1-one (5h). Yield 89\%; M.P. $158-160{ }^{\circ} \mathrm{C}$; IR (KBr, cm-1): 3045 (Ar, C-H ), 1720 (C=0), 1680 (C=0), $1610(\mathrm{C}=\mathrm{C})$; ${ }^{1} \mathrm{H}$ NMR $\left(\mathrm{CDCl}_{3}, \delta / \mathrm{ppm}\right): 8.61$ (s, $\left.1 \mathrm{H}, \mathrm{C}-\mathrm{H}\right), 7.89$ (d, $\left.1 \mathrm{H},=\mathrm{C}-\mathrm{H}\right), 7.78-7.60$ (d, $4 \mathrm{H}$, coumarin), 7.44-7.32 (s, 2H, Ar-H), $6.92(\mathrm{~d}, 1 \mathrm{H},=\mathrm{C}-\mathrm{H})$, 3.94 (s, 9H, $\left.3 \mathrm{OCH}_{3}\right) ;{ }^{13} \mathrm{C}$ NMR $\left(\mathrm{CDCl}_{3}, \delta / \mathrm{ppm}\right): 189.10,162.38$, 161.74, 161.05, 154.78, 147.29, 138.82, 130.04, 127.65, 126.78, 124.64, 124.19, 122.04, 114.66, 105.27, 90.81, 55.64, 55.18; Anal. Calc. for $\mathrm{C}_{21} \mathrm{H}_{18} \mathrm{O}_{6}$ (366.37): C 68.85\%, H 4.95\%; Found: C $68.80 \%, \mathrm{H} 4.91 \%$.

1-(3'-Coumarinyl)-3-(3",5"-dimethoxyphenyl)-2-propen-1one (5i). Yield 88\%; M.P. 147-149 ${ }^{\circ} \mathrm{C}$; IR (KBr, cm-1): $3026(\mathrm{Ar}$, $\mathrm{C}-\mathrm{H}), 1722(\mathrm{C}=0), 1685(\mathrm{C}=0), 1606(\mathrm{C}=\mathrm{C}) ;{ }^{1} \mathrm{H}$ NMR ( $\mathrm{CDCl}_{3}$, $\delta / \mathrm{ppm}): 8.61$ (s, 1H, C-H), $7.89(\mathrm{~d}, 1 \mathrm{H},=\mathrm{C}-\mathrm{H}), 7.78-7.60(\mathrm{~d}, 4 \mathrm{H}$, coumarin), 7.44-7.36 (s, 3H, Ar-H), $6.84(\mathrm{~d}, 1 \mathrm{H},=\mathrm{C}-\mathrm{H}), 3.90$ (s, $\left.6 \mathrm{H}, 2 \mathrm{OCH}_{3}\right) ;{ }^{13} \mathrm{C} \mathrm{NMR}\left(\mathrm{CDCl}_{3}, \delta / \mathrm{ppm}\right): 188.19,162.65,161.45$, 154.84, 147.58, 138.83, 137.33, 130.04, 128.08, 127.69, 124.78, $124.14,122.02,114.74,109.81,100.04,55.63$; Anal. Calc. for $\mathrm{C}_{20} \mathrm{H}_{16} \mathrm{O}_{5}$ (336.34): C 71.42\%, H 4.79\%; Found: C 71.50\%, H $4.63 \%$

1-(3'-Coumarinyl)-3-(4"-dimethylaminophenyl)-2-propen-1one (5j). Yield 89\%; M.P. $108-110^{\circ} \mathrm{C}$; IR $\left(\mathrm{KBr}, \mathrm{cm}^{-1}\right): 3035(\mathrm{Ar}$, $\mathrm{C}-\mathrm{H}), 1714(\mathrm{C}=0), 1681(\mathrm{C}=0), 1606(\mathrm{C}=\mathrm{C}) ;{ }^{1} \mathrm{H}$ NMR $\left(\mathrm{CDCl}_{3}\right.$, $\delta / \mathrm{ppm}): 8.58(\mathrm{~s}, 1 \mathrm{H}, \mathrm{C}-\mathrm{H}), 7.92(\mathrm{~d}, 1 \mathrm{H},=\mathrm{C}-\mathrm{H}), 7.77-7.66(\mathrm{~m}, 4 \mathrm{H}$, coumarin), $7.66(\mathrm{~d}, 2 \mathrm{H}, \mathrm{Ar}-\mathrm{H}), 7.44(\mathrm{~d}, 1 \mathrm{H},=\mathrm{C}-\mathrm{H}), 6.72(\mathrm{~d}, 2 \mathrm{H}$, $\mathrm{Ar}-\mathrm{H}), 3.07$ (s, 6H, 2CH3); ${ }^{13} \mathrm{C}$ NMR $\left(\mathrm{CDCl}_{3}, \delta / \mathrm{ppm}\right): 188.51$, $162.65,154.77,151.10,147.23,138.83,131.29,130.04,128.07$, 127.78, 124.64, 124.54, 123.47, 122.02, 114.71, 112.02, 39.71; Anal. Calc. for $\mathrm{C}_{20} \mathrm{H}_{17} \mathrm{NO}_{3}$ (319.36): C 75.22\%, H 5.37\%, N 4.39\%; Found: C 75.02\%, H 5.29\%, N 4.20\%.

1-(3'-Coumarinyl)-3-(4"-fluorophenyl)-2-propen-1-one (5k). Yield 88\%; M.P. 171-173 ${ }^{\circ} \mathrm{C}$; IR ( $\left.\mathrm{KBr}, \mathrm{cm}^{-1}\right)$ : $3031(\mathrm{Ar}$, $\mathrm{C}-\mathrm{H}$ ), 1718 (C=0), 1666 (C=0), 1612 (C=C); ${ }^{1} \mathrm{H}$ NMR (CDCl 3 , $\delta / \mathrm{ppm}): 8.59(\mathrm{~s}, 1 \mathrm{H}, \mathrm{C}-\mathrm{H}), 7.98(\mathrm{~d}, 1 \mathrm{H},=\mathrm{C}-\mathrm{H}), 7.67-7.52(\mathrm{~m}, 4 \mathrm{H}$, coumarin), 7.41-7.26 (m, 4H, Ar-H), $6.84(\mathrm{~d}, 1 \mathrm{H},=\mathrm{C}-\mathrm{H}) ;{ }^{13} \mathrm{C}$ NMR $\left(\mathrm{CDCl}_{3}, \delta / \mathrm{ppm}\right): 191.50,162.20,154.27,147.47,138.38$, $135.78,134.01,130.78,129.78,129.21,128.07,127.64,124.64$, 124.06, 122.04, 114.60; Anal. Calc. for $\mathrm{C}_{18} \mathrm{H}_{11} \mathrm{O}_{3} \mathrm{~F}$ (295.29): C
73.22\%, H 4.10\%, F 6.43\%; Found: C 73.35\%, H 4.03\%, F $6.29 \%$.

1-(3'-Coumarinyl)-3-(4"-bromophenyl)-2-propen-1-one (5I). Yield 89\%; M.P. 189-191 ${ }^{\circ} \mathrm{C}$; IR $\left(\mathrm{KBr}, \mathrm{cm}^{-1}\right)$ : 3034 (Ar, C-H), 1710 (C=0),1680 (C=0), 1610 (C=C); ${ }^{1} \mathrm{H}$ NMR ( $\mathrm{CDCl}_{3}$, $\delta / \mathrm{ppm}): 8.57$ (s, 1H, C-H), $7.90(\mathrm{~d}, 1 \mathrm{H},=\mathrm{C}-\mathrm{H}), 7.71-7.50(\mathrm{~m}, 4 \mathrm{H}$, coumarin), 7.40-7.16 (m, 4H, Ar-H), 6.59 (d, $1 \mathrm{H},=\mathrm{C}-\mathrm{H}) ;{ }^{13} \mathrm{C}$ NMR ( $\left.\mathrm{CDCl}_{3}, \delta / \mathrm{ppm}\right): 190.44,162.24,154.27,147.47,138.47$, 135.66, 134.04, 130.78, 129.78, 129.21, 128.07, 127.64, 124.64, 124.10, 122.02, 114.60; Anal. Calc. for $\mathrm{C}_{18} \mathrm{H}_{11} \mathrm{O}_{3} \mathrm{Br}$ (356.19): C $60.70 \%$, H 3.40\%, Br 22.43\%; Found: C 60.63\%, H 3.98\%, Br $22.20 \%$.

1-(3'-Coumarinyl)-3-(4"-nitrophenyl)-2-propen-1-one (5m). Yield 89\%; M.P. 154-156 ${ }^{\circ} \mathrm{C}$; IR $\left(\mathrm{KBr}, \mathrm{cm}^{-1}\right)$ : 3045 (Ar, $\mathrm{C}-\mathrm{H}), 1714$ (C=0), 1678 (C=0), $1611(\mathrm{C}=\mathrm{C})$; ${ }^{1} \mathrm{H}$ NMR ( $\mathrm{CDCl}_{3}$, $\delta / \mathrm{ppm}): 8.74(\mathrm{~s}, 1 \mathrm{H}, \mathrm{C}-\mathrm{H}), 7.65(\mathrm{~d}, 1 \mathrm{H},=\mathrm{C}-\mathrm{H}), 7.55-7.48(\mathrm{~m}, 4 \mathrm{H}$, coumarin), 7.37-7.20 (m, 4H, Ar-H), $6.56(\mathrm{~d}, 1 \mathrm{H},=\mathrm{C}-\mathrm{H}) ;{ }^{13} \mathrm{C}$ NMR ( $\left.\mathrm{CDCl}_{3}, \delta / \mathrm{ppm}\right): 190.50,162.20,154.27,147.47,138.38$, 135.78, 134.01, 130.78, 129.78, 129.21, 128.07, 127.64, 124.64, 124.06, 122.04, 114.60; Anal. Calc. for $\mathrm{C}_{18} \mathrm{H}_{11} \mathrm{O}_{5} \mathrm{~N}$ (321.29): C $67.29 \%, \mathrm{H} 3.45 \%$, N 4.36\%; Found: C 67.31\%, H 3.38\%, N $4.22 \%$.

1-(3'-Coumarinyl)-3-(3"-nitrophenyl)-2-propen-1-one (5n). Yield 88\%; M.P. 205-207 ${ }^{\circ} \mathrm{C}$; IR (KBr, cm-1): 3030 (Ar, C-H), $1710(\mathrm{C}=0), 1683(\mathrm{C}=0), 1610(\mathrm{C}=\mathrm{C}) ;{ }^{1} \mathrm{H} \mathrm{NMR}\left(\mathrm{CDCl}_{3}, \delta / \mathrm{ppm}\right)$ : 8.97 (s, 1H, C-H), 7.87 (d, 1H, =C-H), 7.65-7.49 (m, 4H, coumarin), 7.44-7.21 (m, 4H, Ar-H), $6.68(\mathrm{~d}, 1 \mathrm{H},=\mathrm{C}-\mathrm{H}) ;{ }^{13} \mathrm{C} \mathrm{NMR}$ $\left(\mathrm{CDCl}_{3}, \delta / \mathrm{ppm}\right): 188.51,162.65,154.84,149.57,147.25$, 138.89, 138.28, 135.19, 130.88, 130.08, 128.24, 127.67, 125.61, $124.54,122.02,121.64,114.47$; Anal. Calc. for $\mathrm{C}_{18} \mathrm{H}_{11} \mathrm{O}_{5} \mathrm{~N}$ (321.29): C 67.29\%, H 3.45\%, N 4.36\%; Found: C 67.31\%, H $3.38 \%$, N $4.22 \%$.

\subsection{General procedure for the synthesis of pyrazolyl coumarins (7a-7h) [35]}

A mixture of salicylaldehyde $\mathbf{1}(1 \mathrm{mmol})$, methyl acetoacetate or ethyl acetoacetate $\mathbf{2}(1 \mathrm{mmol})$, aromatic aldehyde $\mathbf{3}$ (1 mmol) and hydrazine hydrate $6(1 \mathrm{mmol})$ were mixed in the presence of Bi(OTf) 3 ( $5 \mathrm{~mol} \%)$ in DCM $(5 \mathrm{~mL})$. The reaction mixture was stirred at $50{ }^{\circ} \mathrm{C}$ for the specified time (see Table 3). The progress of the reaction was monitored by TLC. After completion of the reaction, the reaction mixture was allowed to stand overnight. The solid material was filtered off, washed with water, dried, and recrystallized from ethanol to furnish pure pyrazolyl coumarin derivatives.

3-(4,5-Dihydro-5-(4-methoxyphenyl)-1H-pyrazol-3-yl)-2Hchromen-2-one (7a). Yield 90\%; M.P. $183-185{ }^{\circ} \mathrm{C}$; IR (KBr; $\mathrm{cm}^{-1}$ ): 1634 (C=N), 3268 (Ar N-H), 1172 (-OCH3); ${ }^{1} \mathrm{H}$ NMR $\left(\mathrm{CDCl}_{3}, \delta / \mathrm{ppm}\right): 6.72-7.27(\mathrm{~m}, 13 \mathrm{H}, \mathrm{Ar}), 7.46(\mathrm{~s}, 1 \mathrm{H}$, coumarin), 4.80 (d, 1H, 5-H of pyrazoline), 3.301 (d, 1H, 4-Htrans of pyrazoline), $3.305\left(\mathrm{~d}, 1 \mathrm{H}, 4-\mathrm{H}_{\text {cis }}\right.$ of pyrazoline), 3.73 (s, 3H, -OCH3); Anal. Calc. for $\mathrm{C}_{19} \mathrm{H}_{16} \mathrm{O}_{3} \mathrm{~N}_{2}$ : C 71.24\%, N 8.74\%; Found: C $70.68 \%$, N 8.55\%.

3-(4,5-dihydro-5-(2-methoxyphenyl)-1H-pyrazol-3-yl)-2Hchromen-2-one (7b). Yield 88\%; M.P. $166-168{ }^{\circ} \mathrm{C}$; IR (KBr; $\left.\mathrm{cm}^{-1}\right)$ : $1554(\mathrm{C}=\mathrm{N}), 3245(\mathrm{ArN}-\mathrm{H}) ;{ }^{1} \mathrm{H}$ NMR (CDCl $3 / \mathrm{DMSO}-\mathrm{d} 6$, 
$\delta / \mathrm{ppm}): 6.72-7.27(\mathrm{~m}, 13 \mathrm{H}, \mathrm{Ar}), 7.65$ (s, 1H, coumarin), 4.82 (d, $1 \mathrm{H}, 5$ - $\mathrm{H}$ of pyrazoline), $3.23\left(\mathrm{~d}, 1 \mathrm{H}, 4-\mathrm{H}_{\text {trans }}\right.$ of pyrazoline), 3.30 (d, $1 \mathrm{H}, 4-\mathrm{H}_{\text {cis }}$ of pyrazoline), 3.73 (s, $\left.3 \mathrm{H},-\mathrm{OCH} 3\right)$; Anal. Calc. for $\mathrm{C}_{19} \mathrm{H}_{16} \mathrm{O}_{3} \mathrm{~N}_{2}$ : C 71.24\%, N 8.74\%; Found: C 70.85\%, N 8.34\%.

3-(5-(4-Chlorophenyl)-4,5-dihydro-1H-pyrazol-3-yl)-2Hchromen-2-one (7c). Yield 95\%; M.P. 175-177 ${ }^{\circ} \mathrm{C}$; IR (KBr; $\mathrm{cm}^{-1}$ ): 1622 (C=N), 3282 (ArN-H), 1091 (Ar-Cl); ${ }^{1} \mathrm{H}$ NMR ( $\left.\mathrm{CDCl}_{3} / \mathrm{DMSO}-\mathrm{d} 6, \delta / \mathrm{ppm}\right): 7.01-7.28(\mathrm{~m}, 13 \mathrm{H}, \mathrm{Ar}$ ), 7.40 (s, 1H, coumarin), 5.01 (d, $1 \mathrm{H}, 5-\mathrm{H}$ of pyrazoline), 3.30 (d, 1H, 4-H trans of pyrazoline), 3.33 (d, $1 \mathrm{H}, 4-\mathrm{H}_{\text {cis }}$ of pyrazoline); Anal. Calc. for $\mathrm{C}_{18} \mathrm{H}_{13} \mathrm{ClO}_{2} \mathrm{~N}_{2}$ : C 66.57\%, N 8.63\%; Found: C 66.43\%, N 8.27\%.

3-(5-(4-Fluorophenyl)-4,5-dihydro-1H-pyrazol-3-yl)-2Hchromen-2-one (7d): Yield 91\%; M.P. $180-182{ }^{\circ} \mathrm{C}$; IR (KBr; $\mathrm{cm}^{-1}$ ): $1634(\mathrm{C}=\mathrm{N}), 3268$ (Ar N-H), 1042 (Ar C-F); ${ }^{1} \mathrm{H}$ NMR ( $\left.\mathrm{CDCl}_{3} / \mathrm{DMSO}-\mathrm{d} 6, \delta / \mathrm{ppm}\right): 6.813-7.5(\mathrm{~m}, 13 \mathrm{H}, \mathrm{Ar}), 7.49$ (s, $1 \mathrm{H}$, coumarin), 4.86 (d, 1H, 5-H of pyrazoline), 3.25 (d, 1H, 4- $\mathrm{H}_{\text {trans }}$ of pyrazoline), 3.307 (d,1H, 4- $\mathrm{H}_{\text {cis }}$ of pyrazoline); Anal. Calc. for $\mathrm{C}_{18} \mathrm{H}_{13} \mathrm{FO}_{2} \mathrm{~N}_{2}$. C 70.12\%, N 9.09\%; Found: C 69.86\%, N 9.13\%.

3-(5-(4-Bromophenyl)-4,5-dihydro-1H-pyrazol-3-yl)-2Hchromen-2-one (7e). Yield 89\%; M.P. 176-178 ${ }^{\circ} \mathrm{C}$; IR (KBr; $\mathrm{cm}^{-1}$ ): $1623.53(\mathrm{C}=\mathrm{N}), 3257(\mathrm{Ar} \mathrm{N}-\mathrm{H}), 658(\mathrm{C}-\mathrm{Br}) ;{ }^{1} \mathrm{H}$ NMR ( $\left.\mathrm{CDCl}_{3} / \mathrm{DMSO}-\mathrm{d} 6, \delta / \mathrm{ppm}\right): 7.01-7.38(\mathrm{~m}, 13 \mathrm{H}, \mathrm{Ar}), 7.46$ (s, $1 \mathrm{H}$, coumarin), 4.89 (d, 1H, 5-H of pyrazoline), 3.28 (d, 1H, 4- $\mathrm{H}_{\text {trans }}$ of pyrazoline), 3.33 (d, 1H, 4-H $\mathrm{H}_{\text {cis }}$ of pyrazoline); Anal. Calc. for $\mathrm{C}_{18} \mathrm{H}_{13} \mathrm{BrO}_{2} \mathrm{~N}_{2}$. C 58.56\%, N 7.59\%; Found: C 59.02\%, N 7.11\%.

3-(4,5-dihydro-5-(4-nitrophenyl)-1H-pyrazol-3-yl)-2H-chro men-2-one (7f): Yield 86\%; M.P. $199-201{ }^{\circ} \mathrm{C}$; IR (KBr; $\left.\mathrm{cm}^{-1}\right)$ : $1558(\mathrm{C}=\mathrm{N}), 3234(\mathrm{Ar} \mathrm{N}-\mathrm{H}), 1519\left(\right.$ Symmt $=\mathrm{N}-\mathrm{O}$ of $\left.\mathrm{NO}_{2}\right), 1340$ (Asymmt $=\mathrm{N}-\mathrm{O}$ of $\left.\mathrm{NO}_{2}\right) ;{ }^{1} \mathrm{H}$ NMR $\left(\mathrm{CDCl}_{3} / \mathrm{DMSO}-\mathrm{d} 6, \delta / \mathrm{ppm}\right)$ : 7.02-8.14 (m, 13H, Ar), 7.46 (s, 1H, coumarin), $4.86(\mathrm{~d}, 1 \mathrm{H}, 5-\mathrm{H}$ of pyrazoline), 3.301 (d, $1 \mathrm{H}, 4-\mathrm{H}_{\text {trans }}$ of pyrazoline), $3.305(\mathrm{~d}, 1 \mathrm{H}$ $4-\mathrm{H}_{\text {cis }}$ of pyrazoline); Anal. Calc. for $\mathrm{C}_{18} \mathrm{H}_{13} \mathrm{O}_{4} \mathrm{~N}_{3}$ : C $64.47 \%, \mathrm{~N}$ 12.53\%; Found: C $64.30 \%$, N 12.81\%.

3-(4,5-Dihydro-5-(3-nitrophenyl)-1H-pyrazol-3-yl)-2Hchromen-2-one (7g). Yield 87\%; M.P. 189-190 ${ }^{\circ} \mathrm{C}$; IR (KBr; $\left.\mathrm{cm}^{-1}\right)$ : $1622(\mathrm{C}=\mathrm{N}), 3274(\mathrm{Ar} \mathrm{N}-\mathrm{H}), 1488\left(\right.$ Symmt $=\mathrm{N}-\mathrm{O}$ of $\left.\mathrm{NO}_{2}\right)$, 1350 (Asymmt=N-O of $\mathrm{NO}_{2}$ ); ${ }^{1} \mathrm{H}$ NMR ( $\mathrm{CDCl}_{3} / \mathrm{DMSO}-\mathrm{d} 6$, $\delta / \mathrm{ppm}$ ): 6.90-8.07 (m, 13H, Ar), 7.49 (s, 1H, coumarin), 4.86 (d, $1 \mathrm{H}, 5-\mathrm{H}$ of pyrazoline), 3.30 (d, $1 \mathrm{H}, 4-\mathrm{H}_{\text {trans }}$ of pyrazoline), 3.33 (d, $1 \mathrm{H}, 4-\mathrm{H}_{\text {cis }}$ of pyrazoline); Anal. Calc. for $\mathrm{C}_{18} \mathrm{H}_{13} \mathrm{O}_{4} \mathrm{~N}_{3}$ : C 64.47\%, N 12.53\%; Found: C 64.52\%, N 11.98\%.

3-(4,5-Dihydro-5-p-tolyl-1H-pyrazol-3-yl)-2H-chromen-2one (7h). Yield 90\%; M.P. $160-162{ }^{\circ} \mathrm{C}$; IR (KBr; cm$\left.{ }^{-1}\right): 1620$ $(\mathrm{C}=\mathrm{N}), 3284(\mathrm{ArN}-\mathrm{H})$; ${ }^{1} \mathrm{H}$ NMR ( $\left.\mathrm{CDCl}_{3} / \mathrm{DMSO}-\mathrm{d} 6, \delta / \mathrm{ppm}\right)$ : 7.00-7.27 (m, 13H, Ar), 7.42 (s, 1H, coumarin), 4.55 (d, 1H, 5-H of pyrazoline), $3.29\left(\mathrm{~d}, 1 \mathrm{H}, 4-\mathrm{H}_{\text {trans }}\right.$ of pyrazoline), $3.31(\mathrm{~d}, 1 \mathrm{H}$, 4- $\mathrm{H}_{\text {cis }}$ of pyrazoline), $2.35\left(\mathrm{~s}, 3 \mathrm{H}, \mathrm{CH}_{3}\right)$; Anal. Calc. for $\mathrm{C}_{19} \mathrm{H}_{16} \mathrm{O}_{2} \mathrm{~N}_{2} \mathrm{C} 74.98 \%$, N 9.20\%; Found: C 73.84\%, N 8.56\%.

\section{Results and discussion}

\subsection{The reaction conditions}

Initially, the synthesized compounds $\mathbf{5 a - 5 n}$ were obtained from the three-component reaction of salicylaldehyde, an $\alpha$-ketoester (methyl acetoacetate or ethyl acetoacetate) and various aromatic aldehydes in DCM with the addition of a catalytic amount of the green catalyst Bi(OTf) 3 (5 mol\%) (Scheme 1).

The optimization of the reaction conditions was then studied for 5c. No product was obtained from the reaction of salicylaldehyde, ethyl acetoacetate and 4-chlorobenzaldehyde in the absence of the catalyst at $50{ }^{\circ} \mathrm{C}$ in DCM (Table 1, entry 1). The reaction was then performed in the presence of various simple metal complexes such as those of $\mathrm{Fe}, \mathrm{Zn}, \mathrm{Mg}$ and $\mathrm{Cu}$ to examine their catalytic activity (Table 1, entries 2-9). Among these metal salts $(5 \mathrm{~mol} \%), \mathrm{Bi}(\mathrm{OTf})_{3}$ was found to be the most effective catalyst and afforded the desired product $\mathbf{5 c}$ in $96 \%$ yield (Table 1 , entry 11$)$. When using methanol $(\mathrm{MeOH}), N, N$-dimethylformamide (DMF), toluene $\left(\mathrm{PhCH}_{3}\right)$ and acetonitrile $\left(\mathrm{CH}_{3} \mathrm{CN}\right)$ instead of DCM, relatively lower yields were observed (Table 1, entries 16-19). The desired products were rapidly precipitated in good yield on warming for 15-30 min (see Table 2).

Compounds $\mathbf{7 a - 7 h}$ were synthesized using a one-pot four-component reaction between salicylaldehyde, an $\alpha$-ketoester (methyl acetoacetate or ethyl acetoacetate), several aromatic aldehydes and hydrazine hydrate in DCM under the same conditions used for the synthesis of $\mathbf{5}$. The products were

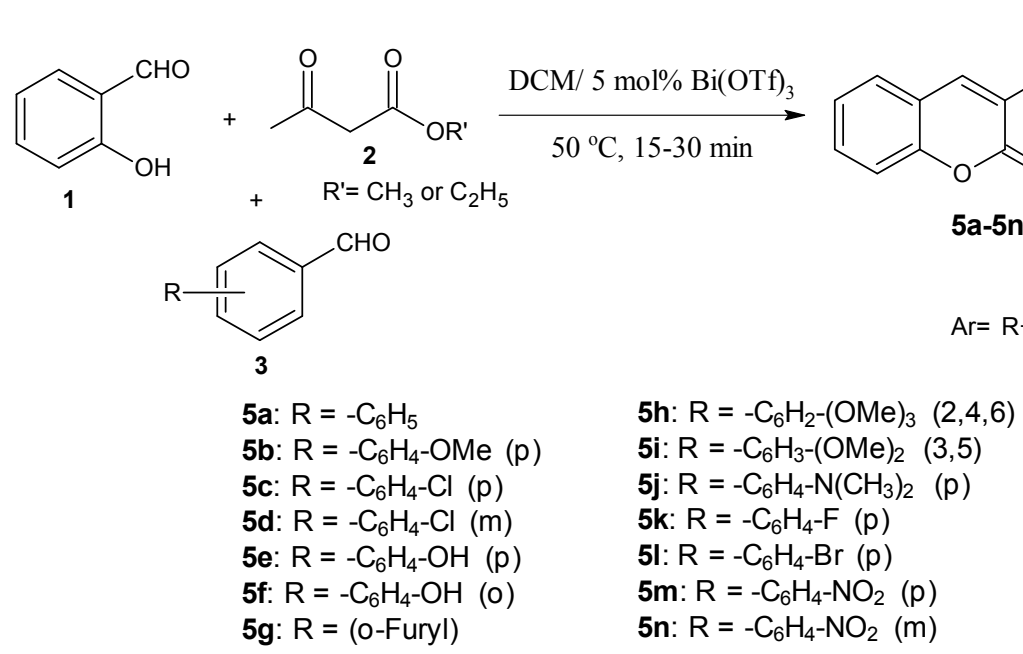

Scheme 1. Synthesis of 3-cinnamoyl coumarin derivatives (5a-5n). 
Table 1

Optimization of the conditions for the synthesis of $\mathbf{5 c}$.

\begin{tabular}{|c|c|c|c|}
\hline Entry & Catalyst (mol\%) & Conditions & Yield* (\%) \\
\hline 1 & No catalyst & $\mathrm{DCM}, 50^{\circ} \mathrm{C}, 6 \mathrm{~h}$ & None \\
\hline 2 & $\mathrm{FeCl}_{3} \cdot 6 \mathrm{H}_{2} \mathrm{O}(5)$ & $\mathrm{DCM}, 50^{\circ} \mathrm{C}, 15 \mathrm{~min}$ & 44 \\
\hline 3 & $\mathrm{Fe}(\mathrm{OTf})_{3}(5)$ & $\mathrm{DCM}, 50^{\circ} \mathrm{C}, 15 \mathrm{~min}$ & 56 \\
\hline 4 & $\mathrm{ZnBr}_{2}(5)$ & $\mathrm{DCM}, 50^{\circ} \mathrm{C}, 15 \mathrm{~min}$ & 51 \\
\hline 5 & $\mathrm{Zn}(\mathrm{OTf})_{2}(5)$ & $\mathrm{DCM}, 50^{\circ} \mathrm{C}, 15 \mathrm{~min}$ & 58 \\
\hline 6 & $\mathrm{MgCl}_{2}(5)$ & $\mathrm{DCM}, 50^{\circ} \mathrm{C}, 15 \mathrm{~min}$ & 50 \\
\hline 7 & $\operatorname{Mg}(\mathrm{OTf})_{2}(5)$ & $\mathrm{DCM}, 50^{\circ} \mathrm{C}, 15 \mathrm{~min}$ & 68 \\
\hline 8 & $\mathrm{CuCl}_{2}(5)$ & $\mathrm{DCM}, 50^{\circ} \mathrm{C}, 15 \mathrm{~min}$ & 54 \\
\hline 9 & $\mathrm{Cu}(\mathrm{OTf})_{2}(5)$ & $\mathrm{DCM}, 50^{\circ} \mathrm{C}, 15 \mathrm{~min}$ & 66 \\
\hline 10 & $\mathrm{Bi}(\mathrm{OTf})_{3}(6)$ & $\mathrm{DCM}, 50^{\circ} \mathrm{C}, 15 \mathrm{~min}$ & 96 \\
\hline 11 & $\mathrm{Bi}(\mathrm{OTf})_{3}(5)$ & $\mathrm{DCM}, 50^{\circ} \mathrm{C}, 15 \mathrm{~min}$ & 96 \\
\hline 12 & $\mathrm{Bi}(\mathrm{OTf})_{3}(4)$ & $\mathrm{DCM}, 50^{\circ} \mathrm{C}, 15 \mathrm{~min}$ & 90 \\
\hline 13 & $\mathrm{Bi}(\mathrm{OTf})_{3}(3)$ & $\mathrm{DCM}, 50^{\circ} \mathrm{C}, 15 \mathrm{~min}$ & 86 \\
\hline 14 & $\mathrm{Bi}(\mathrm{OTf})_{3}(2)$ & $\mathrm{DCM}, 50^{\circ} \mathrm{C}, 15 \mathrm{~min}$ & 73 \\
\hline 15 & $\mathrm{Bi}(\mathrm{OTf})_{3}(1)$ & $\mathrm{DCM}, 50^{\circ} \mathrm{C}, 15 \mathrm{~min}$ & 64 \\
\hline 16 & $\mathrm{Bi}(\mathrm{OTf})_{3}(5)$ & $\mathrm{MeOH}, 50^{\circ} \mathrm{C}, 15 \mathrm{~min}$ & 15 \\
\hline 17 & $\mathrm{Bi}(\mathrm{OTf})_{3}(5)$ & $\mathrm{DMF}, 50^{\circ} \mathrm{C}, 15 \mathrm{~min}$ & 56 \\
\hline 18 & $\mathrm{Bi}(\mathrm{OTf})_{3}(5)$ & $\mathrm{PhCH}_{3}, 50{ }^{\circ} \mathrm{C}, 15 \mathrm{~min}$ & 44 \\
\hline 19 & $\mathrm{Bi}(\mathrm{OTf})_{3}(5)$ & $\mathrm{CH}_{3} \mathrm{CN}, 50^{\circ} \mathrm{C}, 15 \mathrm{~min}$ & 62 \\
\hline
\end{tabular}

Reaction conditions: $\mathbf{1}$ (1 $\mathrm{mmol}), \mathbf{2}(1 \mathrm{mmol}), \mathbf{3 c}(1 \mathrm{mmol})$ and catalyst $(0.05 \mathrm{mmol})$ in solvent $(5 \mathrm{~mL}) .{ }^{*}$ Isolated yields based on $\mathbf{5 c}$.

precipitated after cooling for 2-4 h (see Table 3 and Scheme 2).

To gain further insights into the reaction, products $\mathbf{5 a - 5 n}$ were synthesized in two steps. In the first step, the precursor 3 -acetylcoumarin $\mathbf{4}$ was prepared by a Knoevenagel reaction between salicylaldehyde and an $\alpha$-ketoester (methyl acetoacetate or ethyl acetoacetate) under basic conditions in $89 \%$ yield $[47,48]$. In the second step, 4 was reacted with various aromatic aldehydes to give the final products $\mathbf{5 a - 5 n}$ by a Claisen-Schmidt aldolic condensation. Under the same conditions as those used in the one-pot reaction, these reactions take a long time and have low yields. The same result was also seen for products $\mathbf{7 a - 7 h}$, which were obtained from coumarin-chalcone compounds with hydrazine hydrate (Scheme 3).
Table 2

Reaction time for the synthesis of $\mathbf{5 a - 5 n}$ using a one-pot reaction protocol.

\begin{tabular}{lc|cc}
\hline Compd. & Reaction time (min) & Compd. & Reaction time (min) \\
\hline $\mathbf{5 a}$ & 22 & $\mathbf{5 h}$ & 20 \\
$\mathbf{5 b}$ & 18 & $\mathbf{5 i}$ & 30 \\
$\mathbf{5 c}$ & 15 & $\mathbf{5 j}$ & 30 \\
$\mathbf{5 d}$ & 25 & $\mathbf{5 k}$ & 25 \\
$\mathbf{5 e}$ & 20 & $\mathbf{5 l}$ & 25 \\
$\mathbf{5 f}$ & 20 & $\mathbf{5 m}$ & 30 \\
$\mathbf{5 g}$ & 20 & $\mathbf{5 n}$ & 30 \\
\hline
\end{tabular}

Table 3

Reaction time for the synthesis of $\mathbf{7 a - 7 h}$ using the one-pot reaction protocol.

\begin{tabular}{lc|cc}
\hline Compd. & Reaction time $(\mathrm{h})$ & Compd. & Reaction time $(\mathrm{h})$ \\
\hline $\mathbf{7 a}$ & 3 & $\mathbf{7 e}$ & 4 \\
$\mathbf{7 b}$ & 3 & $\mathbf{7 f}$ & 3 \\
$\mathbf{7 c}$ & 2 & $\mathbf{7 g}$ & 4 \\
$\mathbf{7 d}$ & 2 & $\mathbf{7 h}$ & 4 \\
\hline
\end{tabular}

\subsection{DPPH radical scavenging assay}

The 2,2-diphenyl-1-picrylhydrazyl (DPPH) assay was based on the reported method [49,50]. A rapid, simple and inexpensive method to measure antioxidant capacity of substances involves the use of the free radical, DPPH. DPPH is widely used to test the ability of compounds to act as free radical scavengers or hydrogen donors. Antioxidants tested on DPPH were also found extremely effective in cell systems. This simple test further provides information on the ability of a compound to donate electrons during antioxidant action [51]. The radical scavenging mechanism is based on the transfer of acidic $\mathrm{H}$-atom from the compound to DPPH radical to form DPPH-H.

A DMSO sample of the compounds at $50 \mu \mathrm{g} / \mathrm{mL}$ was diluted to $4 \mathrm{~mL}$ using $\mathrm{MeOH}$. To this, $1 \mathrm{~mL}$ of DPPH solution in $\mathrm{MeOH}$ was added. The mixed solution was incubated at room temperature for $30 \mathrm{~min}$. The absorbance of stable DPPH was deter-<smiles>O=Cc1ccccc1O</smiles>

1<smiles>[R]OC(=O)CC(C)=O</smiles>

$+\quad \mathrm{R}^{\prime}=\mathrm{CH}_{3}$ or $\mathrm{C}_{2} \mathrm{H}_{5}$

$\mathrm{DCM} / 5 \mathrm{~mol} \% \mathrm{Bi}(\mathrm{OTf})_{3}$ $50{ }^{\circ} \mathrm{C}, 2 \mathrm{~h}-4 \mathrm{~h}$<smiles>O=c1oc2ccccc2cc1C1=NNC([Al])C1</smiles>

7a-7h<smiles>[R][X]c1ccc(C=O)cc1</smiles>

$$
\begin{aligned}
& \text { 7a: } \mathrm{R}=4-\mathrm{OCH}_{3} \mathrm{C}_{6} \mathrm{H}_{5} \\
& \text { 7c: } \mathrm{R}=4-\mathrm{CIC}_{6} \mathrm{H}_{5} \\
& \text { 7e: } \mathrm{R}=4-\mathrm{BrC}_{6} \mathrm{H}_{5} \\
& \text { 7g: } \mathrm{R}=3-\mathrm{NO}_{2} \mathrm{C}_{6} \mathrm{H}_{5}
\end{aligned}
$$<smiles>[R]c1ccccc1</smiles>

Scheme 2. Synthesis of coumarins linked to pyrazoline. 
<smiles>O=Cc1cc2ccccc2oc1=O</smiles><smiles>[13CH3]/C=C/C(=O)c1cc2ccccc2oc1=O</smiles><smiles>[R]c1cccc(C=O)c1</smiles>

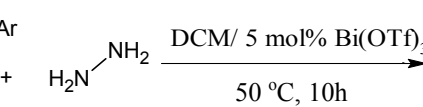
$50^{\circ} \mathrm{C}, 10 \mathrm{~h}$

$\mathrm{CHO}$<smiles>O=[131I]/C=C/C(=O)c1cc2ccccc2oc1=O</smiles><smiles>O=c1oc2ccccc2cc1C1=NNC(Br)C1</smiles><smiles>[R]c1cccc(C=O)c1</smiles>

Scheme 3. Control experiments for the synthesis of $\mathbf{5 c}$ and $\mathbf{7 c}$ in a stepwise reaction.

mined at $517 \mathrm{~nm}$ using UV/Vis spectroscopy, and the remaining amount of DPPH was calculated. Ascorbic acid $(50 \mu \mathrm{g} / \mathrm{mL})$ was used as a standard. The free radical scavenging activity was expressed as follows:

DPPH scavenging activity $(\%)=\left[A_{\mathrm{c}}-A_{\mathrm{s}}\right] /\left[A_{\mathrm{c}}-A_{\mathrm{b}}\right] \times 100$ where $A_{\mathrm{c}}, A_{\mathrm{s}}$ and $A_{\mathrm{b}}$ are the absorbance of the control, the sample and the blank (MeOH+DMSO), respectively. Each sample was assayed at $50 \mu \mathrm{g} / \mathrm{mL}$ and all experiments were carried out in triplicate. The radical scavenging activity is shown in Table 4.

\section{Conclusions}

The synthesis of potential bio-active hybrid molecules obtained from naturally occurring coumarin-chalcone moieties and coumarins linked to pyrazoline using Bi(OTf) 3 as a highly efficient catalyst has been achieved using a one-pot multi-component reaction protocol. This method has the advantages of low cost, short reaction time, high atom economy, convenience and efficiency. It also offers a selective approach to the synthesis of coumarins, a structural motif found in a large number of natural products, pharmaceuticals and functionalized materials. Such a selective procedure offers energy saving advantages and reduces the expense of the purification process. The synthesized compounds showed DPPH scavenging activity. Applications of this protocol to the selective synthesis of bioactive molecules are in progress in our research group.

\section{Table 4}

DPPH radical scavenging assay of compounds $\mathbf{7 a - 7 h}$

\begin{tabular}{ll}
\hline Compound & DPPH scavenging (\%) \\
\hline $\mathbf{7 a}$ & $44.85 \pm 0.32$ \\
$\mathbf{7 b}$ & $59.29 \pm 0.24$ \\
$\mathbf{7 c}$ & $36.56 \pm 0.37$ \\
$\mathbf{7 d}$ & $78.10 \pm 0.42$ \\
$\mathbf{7 e}$ & $51.23 \pm 0.45$ \\
$\mathbf{7 f}$ & $28.57 \pm 0.31$ \\
$\mathbf{7 g}$ & $56.42 \pm 0.32$ \\
$\mathbf{7 h}$ & $27.30 \pm 0.34$ \\
Ascorbic acid & $94.56 \pm 0.34$ \\
\hline
\end{tabular}

\section{Acknowledgments}

The author expresses his deep gratitude to Sohag University in Egypt for supporting and facilitating this study.

\section{References}

[1] Sethna S M, Shah N M. Chem Rev, 1945, 36: 1

[2] Riveiro M E, De Kimpe N, Moglioni A, Vazquez R, Monczor F, Shayo C, Davio C. Curr Med Chem, 2010, 17: 1325

[3] Riveiro M E, Maes D, Vazquez R, Vermeulen M, Mangelinckx S, Jacobs J, Debenedetti S, Shayo C, De Kimpe N, Davio C. Bioorg Med Chem, 2009, 17: 6547

[4] O'Kennedy R, Thornes R D. Coumarins: Biology, Applications, and Mode of Action. Chichester (UK): Wiley, 1997

[5] Yu D L, Suzuki M, Xie L, Morris-Natschke S L, Lee K H. Med Res Rev, 2003, 23: 322

[6] Zhang Q Y, Qin L P, He W D, Van Puywelde L, Maes D, Adams A, De Kimpe N. Planta Medica, 2007, 73: 13

[7] Riveiro M E, Shayo C, Monczor F, Fernandez N, Baldi A, De Kimpe N, Rossi J, Debenedetti S, Davio C. Cancer Lett, 2004, 210: 179

[8] Murray R D H, Mendez J, Brown S A. The Natural Coumarins: Occurrence, Chemistry, and Biochemistry. Chichester (UK): Wiley, 1982

[9] Nguyen V T, Debenedetti S, De Kimpe N. Tetrahedron Lett, 2003, 44: 4199

[10] Maes D, Vervisch S, Debenedetti S, Davio C. Mangelinckx S, Giubellina N, De Kimpe N. Tetrahedron, 2005, 61: 2505

[11] Ngameni B, Watchueng J, Boyom F F, Keumedjio F, Ngadjui B T, Gut J, Abegaz B M, Rosenthal P J. ARKIVOC, 2007: 116

[12] Okwu D E, Ukanwa N. Chem Sin, 2010, 1(2): 21

[13] Ballesteros J F, Sanz M J, Ubeda A, Miranda M A, Iborra S, Paya M, Alcaraz M. J Med Chem, 1995, 38: 2794

[14] Go M L, Wu X, Liu X L. Curr Med Chem, 2005, 12: 483

[15] Mukherjee S, Kumar V, Prasad A K, Raj H G, Bracke M E, Olsen C E, Jain S C, Parmar V S. Bioorg Med Chem, 2001, 9: 337

[16] Liu M, Wilairat P, Croft S L, Tan A L G, Go M L. Bioorg Med Chem, 2003, 11: 2729

[17] Sivakumar P M, Babu S K G, Mukesh D. Chem Pharm Bull, 2007, 55: 44

[18] Viana G S B, Bandeira M A M, Mantos F J A. Phytomedicine, 2003, 10: 189 


\section{Graphical Abstract}

Chin. J. Catal., 2015, 36: 1124-1130 doi: 10.1016/S1872-2067(14)60308-9

\section{Bismuth triflate: A highly efficient catalyst for the synthesis of bio-active coumarin compounds via one-pot multi-component reaction}

Mahmoud. Abd El Aleem. Ali. Ali. El-Remaily*

Sohag University- 82524, Sohag, Egypt

A series of coumarin-chalcone hybrid compounds and coumarins linked to pyrazoline was synthesized in good yields and short time using a simple and efficient method. This method involved the one-pot reaction of salicylaldehyde, an $\alpha$-ketoester and an aromatic aldehyde (in the case of the coumarin-chalcone derivatives) in addition to hydrazine hydrate (in the case of the pyrazolyl coumarins) in the presence of a catalytic amount of bismuth triflate [Bi(OTf) 3,5 mol\%]. The synthesized compounds showed scavenging activity towards the free radical 2,2-diphenyl-1-picrylhydrazyl. All compounds were characterized by IR, ${ }^{1} \mathrm{H}$ NMR and ${ }^{13} \mathrm{C}$ NMR spectroscopy.

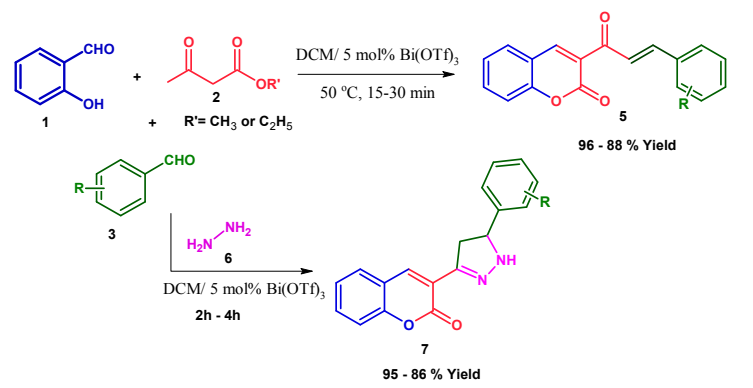

[19] Xia Y, Yang Z Y, Xia P, Bastow K F, Nakanishi Y, Lee K H. Bioorg Med Chem lett, 2000, 10: 699

[20] Ducki S, Forrest R, Hadfield J A, Kendall A, Lawrence N J, McGown A T, Rennison D. Bioorg Med Chem Lett, 1998, 8: 1051

[21] Wagner B D. Molecules, 2009, 14: 210

[22] Dorlars A, Schellhammer C W, Schroeder J. Angew Chem Int Ed, 1975, 14: 665

[23] Kumar S, Bawa S, Drabu S, Kumar R, Gupta H. Recent Patents on Anti-Infective Drug Discovery, 2009, 4: 154

[24] Zhu J P, Bienayme H. Multicomponent Reactions. Weinheim: WILEY-VCH, 2005

[25] Tejedor D, Garcia-Tellado F. Chem Soc Rev, 2007, 36: 484

[26] Ugi I. Pure Appl Chem, 2001, 73: 187

[27] Lieby-Muller F, Simon C, Constantieux T, Rodriguez, J. QSAR Comb Sci, 2006, 25: 432

[28] Trost B M. Angew Chem Int Ed, 1995, 34: 259

[29] Wender P A, Handy S T, Wright D L. Chem Ind, 1997: 767

[30] Weber L. Curr Opin Chem Biol, 2000, 4: 295-302

[31] El-Remaily M A A. Tetrahedron, 2014, 70: 2971

[32] Domling A. Curr Opin Chem Biol, 2002, 6: 306

[33] Leonard N M, Wieland L C, Mohan R S. Tetrahedron, 2002, 58: 8373

[34] Thirupathi P, Kim S S. Tetrahedron, 2009, 65: 5168

[35] Rivera S, Bandyopadhyay D, Banik B K. Tetrahedron Lett, 2009, 50 5445
[36] Antoniotti S, Dunach E. C R Chim, 2004, 7: 679

[37] Mohammadpoor-Baltork I, Khosropour A R, Moghadam M, Tangestaninejad S, Mirkhani V, Baghersad S, Mirjafari A. C R Chim, 2011, 14: 944

[38] Wang Z Z, Fang S Y. Eur J Org Chem, 2009: 5505

[39] Ollevier T. Org Biomol Chem, 2013, 11: 2740

[40] Ollevier T. Top Curr Chem, 2012, 311: 69

[41] Pin F, Comesse S, Garrigues B, Marchaln S, Daich A. J Org Chem, 2007, 72: 1181

[42] Anzalone P W, Baru A R, Danielson E M, Hayes P D, Nguyen M P, Panico A F, Smith R C, Mohan R S.J Org Chem, 2005, 70: 2091

[43] Rueping M, Nachtsheim B J, Ieawsuwan W. Adv Synth Catal, 2006, 348: 1033

[44] Ollevier T, Li Z Y. Adv Synth Catal, 2009, 351: 3251

[45] Venkat Narsaiah A, Reddy B V S, Premalatha K, Reddy S S, Yadav J S. Catal Lett, 2009, 131: 480

[46] Podgorski D M, Krabbe S W, Le L N, Sierszulski P R, Mohan R S. Synthesis, 2010: 2771

[47] Sugino T, Tanaka K. Chem Lett, 2001, 30: 110

[48] Bhatnagar A, Sharma P K, Kumar N, Upadhyay A. Pharm Chem J, 2012, 46: 482

[49] Blois M S. Nature, 1958, 181: 1199

[50] Samshuddin S, Narayana B, Sarojini B K, Yathirajan H S, Raghavendra R. Pharm Chem, 2012, 4: 1445

[51] Tiwari A K. Curr Sci, 2004, 86: 1092 